Published in final edited form as:

Sci Transl Med. 2019 June 12; 11(496): . doi:10.1126/scitranslmed.aav1892.

\title{
The gut microbiota regulates white adipose tissue inflammation and obesity via a family of microRNAs
}

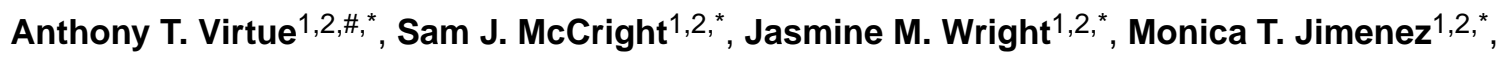 \\ Walter K. Mowel $^{1,2}$, Jonathan J. Kotzin ${ }^{1,2}$, Leonel Joannas ${ }^{1,2}$, Megha G. Basavappa ${ }^{1,2,3}$, \\ Sean P. Spencer ${ }^{1,2}$, Megan L. Clark ${ }^{1,2}$, Stephen H. Eisennagel ${ }^{4}$, Adam Williams ${ }^{5}$, Maayan \\ Levy $^{2,3,6}$, Sasikanth Manne ${ }^{7}$, Sarah E. Henrickson ${ }^{2,8}$, E. John Wherry ${ }^{2,7,9}$, Christoph A. \\ Thaiss $^{2,3,6}$, Eran Elinav ${ }^{6}$, Jorge Henao-Mejia ${ }^{1,2,10}$ \\ ${ }^{1}$ Department of Pathology and Laboratory Medicine, University of Pennsylvania, Philadelphia, PA \\ 19104, USA. \\ ${ }^{2}$ Institute for Immunology, Perelman School of Medicine, University of Pennsylvania, Philadelphia, \\ PA 19104, USA. \\ ${ }^{3}$ Department of Microbiology, University of Pennsylvania, Philadelphia, PA 19104, USA. \\ ${ }^{4}$ Core DMPK, GlaxoSmithKline, Collegeville, PA 19426, USA. \\ ${ }^{5}$ The Jackson Laboratory for Genomic Medicine, Department of Genetics and Genome Sciences, \\ University of Connecticut Health Center, Farmington, CT, 06032, USA. \\ ${ }^{6}$ Department of Immunology, Weizmann Institute of Science, Rehovot, Israel. \\ ${ }^{7}$ Department of Systems Pharmacology and Translational Therapeutics, Perelman School of \\ Medicine, Philadelphia, PA 19104, USA. \\ ${ }^{8}$ Division of Allergy Immunology, The Children's Hospital of Philadelphia, Philadelphia, PA 19104, \\ USA.
}

${ }^{9}$ Parker Institute for Cancer Immunotherapy, Perelman School of Medicine, University of Pennsylvania, Philadelphia, PA 19104, USA.

\footnotetext{
Corresponding author: Jorge Henao-Mejia, jhena@pennmedicine.upenn.edu.

\#Current address: Target Incubator DPU, GlaxoSmithKline, Collegeville, Pennsylvania 19426, USA.

* These authors contributed equally

AUTHOR CONTRIBUTIONS

A.T.V., S.J.M., J.M.W., M.T.J., and J.H.M. designed all experiments. J.H.M. supervised the project. A.T.V., S.J.M., J.M.W., M.T.J., and J.H.M. wrote the manuscript. W.K.M performed flow cytometry analysis of adipose tissue. J.J.K. performed next-generation sequencing and RNA-seq analysis. L.J., S.P.S., M.G.B., M.L.C., and A.W. assisted in in vivo experiments. M.L., C.A.T., and E.E. performed cecal metabolomics analysis. S.H.E. performed all targeted mass spectrometry analysis in mouse plasma. S.M., S.E.C., S.E.H., E.J.W. collected samples from pediatric cohort and performed statistical analysis for indole levels in plasma. Body weight and fat mass measurements, GTT, ITT, gene expression profiling in vitro and in vivo were conducted by A.T.V., S.J.M., J.M.W., M.T.J., and J.H-M.

DATA and MATERIALS AVAILABILITY

All data associated with the manuscript are present in the main text or supplementary materials. Next-generation sequencing data were deposited in the NCBI Gene Expression Omnibus (GSE126135). Data used to generate figures are in data file S1.

COMPETING INTERESTS

E.J.W. has consulting agreements with and is on the scientific advisory boards for Merck, Roche, Pieris, Elstar, and Surface Oncology. E.J.W. has a patent licensing agreement on the PD-1 pathway with Roche/Genentech: "Methods and Compositions for the treatment of persistent infections and cancer by inhibiting the programmed cell death (PD-1) pathway", US patent number 14/144,304. S. E. H. has advised for Horizon Pharma.
} 
${ }^{10}$ Division of Protective Immunity, Department of Pathology and Laboratory Medicine, Children's Hospital of Philadelphia, University of Pennsylvania, Philadelphia, PA 19104, USA.

\section{Abstract}

The gut microbiota is a key environmental determinant of mammalian metabolism. Regulation of white adipose tissue (WAT) by the gut microbiota is a process critical to maintaining metabolic fitness, and gut dysbiosis can contribute to the development of obesity and insulin resistance (IR). However, how the gut microbiota regulates WAT function remains largely unknown. Here, we show that tryptophan-derived metabolites produced by the gut microbiota controlled the expression of the miR-181 family in white adipocytes in mice to regulate energy expenditure and insulin sensitivity. Moreover, we show that dysregulation of the gut microbiota-miR-181 axis was required for the development of obesity, IR, and WAT inflammation in mice. Our results indicate that regulation of miR-181 in WAT by gut microbiota-derived metabolites is a central mechanism by which host metabolism is tuned in response to dietary and environmental changes. As we also found that MIR-181 expression in WAT and the plasma abundance of tryptophan-derived metabolites were dysregulated in a cohort of obese human children, the MIR-181 family may represent a potential therapeutic target to modulate WAT function in the context of obesity.

\section{One-sentence summary:}

Tryptophan-derived metabolites from the gut microbiota control miR-181 expression in mouse white adipocytes to regulate metabolism and inflammation.

\section{INTRODUCTION}

The gut microbiota is a key environmental factor that can contribute to the development of obesity, insulin resistance (IR), and associated pathologies (1). Yet, how the gut microbiota regulates the functions and inflammatory status of central metabolic organs during these disease processes remains poorly understood. White adipose tissue (WAT) is the major site of energy storage in vertebrates and tightly controls glucose homeostasis in mammals (1). WAT expansion, dysfunction, and inflammation are hallmarks of obesity and play a critical role in the development of highly-prevalent metabolic disorders such as IR, atherosclerosis, and non-alcoholic fatty liver disease (2). In recent years, the gut microbiota has emerged as a central regulator of mammalian adipose tissue. The gut microbiota largely promotes energy storage by decreasing thermogenesis in induced brown adipose tissue (iBAT) and promoting WAT expansion (3). Moreover, dysbiosis of the gut microbiota during nutritional surplus contributes to the development of obesity and related disorders $(1,2,4)$. The microbiota modulates the production and function of iBAT by regulating the inflammatory milieu of this tissue. However, the signals from the microbiota that control WAT functions and inflammation, and the molecular mechanisms by which the microbiota regulates this central metabolic organ are largely undetermined (1, 3-6).

The intestinal microbiota produces thousands of small, diffusible metabolites capable of modulating basic physiological processes in the intestine and distant tissues (7). Indeed, some of these metabolites have been shown to regulate metabolic and inflammatory 
processes involved in the pathogenesis of obesity-associated disorders $(1,8)$. However, the functions of most of these metabolites, and how disturbances in metabolite abundance contribute to obesity and associated pathologies, are poorly understood. Moreover, although it is now clear that WAT mass, function, and inflammation are regulated by the gut microbiota, the role of microbiota-derived metabolites in these processes remains mostly unknown.

Accumulating evidence indicates that many microRNAs (miRNAs) have evolved to act not only as genetic switches of specific, individual genes, but also as control nodes in large gene-regulatory networks (9). As such, miRNAs can tune large gene expression programs in response to specific cellular and organismal stimuli in a rapid, reversible manner. Several highly-conserved miRNAs have been shown to play critical roles in controlling metabolic homeostasis, and their dysregulation contributes to the development of obesity and IR (10, 11). However, whether specific miRNAs regulate the function of central metabolic organs in response to microbiota-derived cues is not clear.

The miR-181 family of miRNAs is highly-conserved in metazoans. Although it has been reported that this miRNA family controls inflammatory pathways contributing to the progression of obesity-associated pathologies (12), its role in regulating metabolism and inflammation in response to dietary or environmental changes is largely unknown. Herein, we show that specific microbiota-derived metabolites regulate the expression of $m i R-181$ in white adipocytes to tightly control energy expenditure, adiposity, WAT inflammation, and insulin sensitivity. Moreover, we show that dysregulation of the microbiota-miR-181 axis is required for the development of obesity, IR, and WAT inflammation in mice. Our results indicate that the regulation of miRNAs in central metabolic organs by microbiota-derived cues may be a key mechanism by which host metabolism is tuned in response to dietary and environmental changes.

\section{RESULTS}

\section{The miR-181 family is elevated in WAT of obese mice and humans}

The miR-181 family is composed of 6 highly-conserved mature miRNAs transcribed from three independently-segregating clusters expressed primarily in hematopoietic cells (Fig. 1A, fig. S1A) (13). To begin interrogating the role of the $m i R-181$ family in metabolic regulation, we measured the expression of each miRNA cluster in different tissues from lean mice fed a normocaloric diet (NCD) and obese mice fed a high-fat diet (HFD). All miR-181 clusters were up-regulated in epididymal white adipose tissue (eWAT) during diet-induced obesity (DIO), with no changes in expression detected in other tissues examined (Fig. 1BD). The MIR-181A2-B2 cluster was likewise strongly up-regulated in adipose tissue from obese adult humans (Fig. 1E), indicating that dysregulation of miR-181 in adipose tissue during DIO is conserved in humans. These results suggest that the miR-181 family may play a critical role in the regulation of WAT, and that its dysregulation during DIO could contribute to the pathogenesis of obesity and associated disorders. 


\section{The $m i R-181$ family promotes the progression of DIO and fat mass gain}

Combined ablation of all three miR-181 clusters is embryonic lethal (13). Therefore, to interrogate the role of this miRNA during DIO, we generated mice lacking the two most abundantly-expressed miR-181 clusters ( $m i R-181 a 1-b 1^{-1-} ; m i R-181 a 2-b 2^{-1-}$ ), herein referred to as double knockout (DKO) mice (13). We then fed wild-type (WT) and DKO mice a NCD or HFD and determined their body composition and change in body weight over time. When fed a NCD, WT and DKO mice did not show differences in body weight, but DKO mice had a mild yet significant $(\mathrm{p}=0.008)$ reduction in total fat mass (Fig. $1 \mathrm{~F}-\mathrm{G}$, fig. S1B-D). When administered a HFD, miR-181-deficient mice were completely protected from developing obesity concurrent with a substantially lower total fat mass compared to WT mice (Fig. 1F-G, fig. S1B-D). Furthermore, histological examination of eWAT revealed that DKO mice had a reduction in adipocyte size compared to WT mice (Fig. 1H, fig. S1EG). As we did not observe alterations in lean mass (Fig. 1G, fig. S1C), these results indicate that the $m i R-181$ family plays a critical role in promoting fat mass gain, driving the progression of obesity.

Differences in body weight and composition are the result of alterations in either caloric intake or energy expenditure (14). To understand how the miR-181 family regulates body weight and composition, we measured whole-body metabolism using a Comprehensive Lab Animal Monitoring System (CLAMS) in HFD-fed WT and DKO mice before body weights diverged (fig. S2A). After 6 weeks of HFD feeding, we observed that $\mathrm{O}_{2}$ consumption $\left(\mathrm{VO}_{2}\right), \mathrm{CO}_{2}$ production $\left(\mathrm{VCO}_{2}\right)$, and calculated heat production were elevated in miR-181deficient mice (Fig. 1I-K, fig. S2B-D). Additionally, DKO mice showed an increase in the respiratory exchange ratio (RER) (Fig. 1L, fig. S2E), indicating that $m i R$-181-deficient mice metabolized a mix of carbohydrates and fatty acids for energy. The increased energy expenditure and alterations in substrate utilization in DKO mice were observed without changes in locomotor activity, and with increased food intake (fig. S2F-K). We found similar differences in CLAMS studies performed in the absence of nutritional stress in NCD-fed mice (fig. S3A-J). We did not observe alterations in nutrient absorption in DKO mice (fig. S3K-L). Using general guidelines for interpreting mouse metabolic studies (15), these results suggested that decreased fat mass and resistance to DIO in miR-181-deficient mice were the consequence of increased energy expenditure. To determine whether the increased energy expenditure in $m i R$-181-deficient mice was associated with increased beiging of inguinal WAT (iWAT), we performed 6- and 24-hour cold challenges in WT and DKO mice. We observed moderate increases in metabolic and transcriptional indicators of cold-induced thermogenesis in DKO mice compared to WT mice (fig. S4A-K), consistent with our findings that miR-181 was not induced in iWAT or brown adipose tissue (BAT) during DIO (fig. S4L-M). These results suggested that energy expenditure, which can protect against obesity, was increased in miR-181-deficient animals through multiple mechanisms, some of which may be dependent on Ucp1-mediated thermogenesis. Altogether, these results suggest that aberrantly-elevated $m i R-181$ expression in visceral WAT during nutritional surplus in mice and humans is critical for the progression of DIO and associated disorders. 
miR-181 promotes the development of IR and WAT inflammation

In addition to modulating weight, eWAT has been shown to play a critical role in regulating glucose homeostasis (16), with eWAT inflammation contributing to the development of obesity-induced IR. To establish whether the miR-181 family regulates insulin sensitivity at steady state and during DIO, we measured blood insulin concentrations and performed glucose and insulin tolerance tests in lean and obese WT and DKO mice (Fig. 2A-D). Even without weight differences, lean miR-181-deficient mice were more sensitive to insulin, had greatly reduced steady-state blood insulin concentrations, and scored lower on the homeostatic model assessment-insulin resistance (HOMA-IR) index compared to lean WT mice (Fig. 2A-D, Fig. 2I-J, fig. S5A-B). Moreover, miR-181-deficient mice were protected against obesity-induced IR (Fig. 2E-J, fig. S5A), further supporting a central role for the miR-181 family in modulating insulin sensitivity. Finally, to determine whether eWAT was the primary tissue in which insulin signaling was altered in DKO mice, we evaluated PI3K pathway activity in different tissues upon insulin stimulation in vivo. In agreement with our previous results, we observed an increase in insulin signaling in eWAT from DKO mice relative to WT mice (Fig. 2K, fig. S5C), but not in other tissues, including liver (fig. S5DE). These results suggest that the $m i R-181$ family regulates insulin sensitivity primarily through its activity in WAT.

Obesity is associated with infiltration of inflammatory cells (CD4+ T cells, M1 macrophages, natural killer [NK] cells and group 1 innate lymphoid cells [ILC1s]) into eWAT (17-20), promoting a pro-inflammatory state that contributes to the development of IR (21). Conversely, eWAT from lean animals and humans contains high numbers of cells associated with Type-2 immune responses (T-regulatory cells [Tregs], eosinophils, group 2 innate lymphoid cells [ILC2s], and M2 macrophages), which promote insulin sensitivity (19, $22-24)$. Thus, to determine whether the $m i R-181$ family regulates eWAT inflammation, we performed flow cytometric analysis of immune cell populations from this tissue in HFD-fed WT and DKO animals. In concordance with our functional and metabolic data, eWAT from DKO mice contained more Tregs, eosinophils, and ILC2s (Fig. 2L-M, fig. S5F). Furthermore, expression of several genes associated with M2 macrophage polarization was increased in miR-181-deficient eWAT (Ym1, Relma, Pecr, Mertk, Mrc1, Glul, Fert1, Camk1, Arg1), whereas expression of genes associated with pro-inflammatory M1 macrophage polarization was reduced (Tnfa, Itgax) (Fig. 2N). Finally, there were no differences in immune cell infiltration in eWAT from NCD-fed WT mice compared to DKO mice (fig. S5G). These results suggest that in the context of NCD feeding, miR-181 does not regulate the inflammatory state of eWAT, and that up-regulation of the miR-181 family in eWAT during DIO is required for the induction of a pro-inflammatory state in this tissue, contributing to the development of IR and fat gain.

We next sought to unravel the mechanisms by which the miR-181 family regulates weight and glucose homeostasis. Growing evidence indicates that in many cases, miRNAs evolved to exert their biological function by modulating the coordinated expression of large gene networks (9). This process allows organisms to rapidly tune global gene expression programs without transcriptional or epigenetic alterations. To identify genes regulated by miR-181 in eWAT that control weight gain, adiposity, inflammation, or insulin sensitivity, 
we performed next-generation sequencing of RNA isolated from eWAT of HFD-fed WT and DKO mice. We first asked whether predicted miR-181 target genes were differentially expressed in eWAT from HFD-fed WT and DKO mice. Indeed, we found that predicted miR-181 targets were enriched in eWAT from DKO mice compared to WT controls (Fig. $3 \mathrm{~A}$ ). Several of the most statistically significant (false discovery rate $[\mathrm{FDR}]<0.05$ ) upregulated miR-181 target genes in eWAT from DKO mice have been reported to play critical roles in adipose tissue development (KIf15, Hoxc8, Cbx 7, Esr1, Negr1, Per2, Irs2) (25-30). Furthermore, a subset of these target genes have been shown to regulate body weight (Timp3, Fam73b, Klf15, Cbx 7, Esr1, Irs2) (27, 28, 31-33), and insulin signaling (Irs2, Ano1, Sox6, Klf15, Cbx 7, Esr1, Negr1, Per2) (26-30, 33-35) (Fig. 3A). As increased expression of a number of these genes in eWAT can modulate the development of obesity and IR $(26,27,29,30,33-35)$, we investigated the downstream consequences of increased expression of these miR-181 target genes in DKO mice. Concordant with our previous results (Fig. 2L-N), we found that expression of several pathways associated with inflammatory responses were down-regulated in miR-181-deficient eWAT (Fig. 3B, fig. $\mathrm{S} 5 \mathrm{H}-\mathrm{I}$ ), whereas genes associated with oxidative phosphorylation were enriched in eWAT from DKO mice (Fig. 3B). Taken together, these results suggest that the $m i R-181$ family directly regulates a network of genes controlling adipocyte function and development, energy expenditure, and insulin signaling in WAT.

Our gene expression analysis suggested that $m i R-181$ exerted its primary regulatory activity in WAT adipocytes. However, a range of complex interactions between adipocytes and immune populations within WAT can influence the rate of fat gain and insulin sensitivity (36). To formally establish the cell type within WAT in which $m i R-181$ regulates insulin sensitivity and obesity, we generated four triple conditional knockout (TcKO) mouse strains in which the entire $m i R-181$ family was ablated either in adipocytes or in one of the three major immune cell lineages known to infiltrate WAT: macrophages, CD4+ T cells, and Tregulatory cells. We also generated a $m i R-181 \mathrm{TcKO}$ strain in hepatocytes, another major metabolic cell type, to further assess the tissue-specific function of this miRNA family. We did not observe a decrease in insulin resistance or obesity in mice lacking miR-181 in any of the immune cell subsets or hepatocytes during HFD feeding (Fig. 3C-F, fig. S6A-L). In contrast, deletion of $m i R-181$ in adipocytes resulted in a decrease in weight gain and IR (Fig. 3G-J), largely recapitulating the phenotype of DKO mice. As insulin potently suppresses adipocyte hormone-sensitive lipase, postprandial free fatty acid (FFA) concentrations in plasma measured after a fasting period can accurately predict insulin sensitivity in WAT (37). In agreement with our evaluation of PI3K activation after insulin stimulation (Fig. 2K, fig. S5C-E) and our transcriptomic and genetic analysis (Fig. 3A-B, G-J), we observed that NCD-fed DKO mice had lower postprandial plasma FFA and glucose concentrations after fasting, relative to WT mice (Fig. 3K, fig. S6M). These results, combined with our data indicating that $m i R-181$ does not regulate WAT inflammation during NCD feeding, further support a crucial role for this microRNA family specifically in white adipocytes (Fig. 3K). Adipose tissue can be separated by enzymatic digestion and centrifugation into an adipocyte fraction and a stromal-vascular fraction containing WATresident immune cells (38). By this method we observed an increase in $m i R-181$ expression in adipocytes, but not stromal-vascular cells, upon HFD feeding (fig. S6N). Altogether, these 
results indicate that $m i R$ - 181 expression in adipocytes promotes IR, adiposity, and weight gain in the context of obesity, independent of any potential regulatory roles in other tissues or cell types.

\section{The gut microbiota regulates the expression of the $m i R-181$ family in white adipocytes}

We then sought to investigate the upstream factors regulating miR-181 in WAT. Cytokines are central regulators of WAT function (39), and it is now well-recognized that elevated proinflammatory cytokines promote IR and obesity (40). We hypothesized that cytokines tightly control adipocyte miR-181 expression to regulate WAT inflammation and whole-body glucose and energy homeostasis. To test this hypothesis, we first assessed whether cytokines known to regulate insulin sensitivity and obesity control expression of $m i R-181$ in cultured adipocytes. However, we did not observe changes in miR-181 expression in adipocytes stimulated with pro- or anti-inflammatory cytokines (fig. S7A-D). LPS, a major component of Gram-negative bacterial membranes, potently induces pro-inflammatory cytokines, IR, and WAT inflammation. To test its effect on adipocyte miR-181 expression in vivo, we administered LPS to mice either orally or by intraperitoneal injection. Consistent with our in vitro data, LPS administration did not impact expression of mature miR-181a or miR-181b in adipocytes in vivo (fig. S7E-F). These results indicate that neither inflammatory cytokines nor translocation of LPS are the main regulators of $m i R-181$ expression in adipocytes in vivo.

It is now well-recognized that the gut microbiota is a central regulator of WAT in healthy organisms and promotes WAT dysfunction during DIO $(3,4)$. Furthermore, microbiotaderived signals have been shown to regulate metabolism, and dysbiosis can promote obesity and associated disorders $(1,41)$. Therefore, we aimed to establish whether the microbiota regulates $m i R$ - 181 in adipocytes by comparing $m i R$ - 181 expression in eWAT from germ-free (GF) and conventionally-housed specific pathogen-free (SPF) mice. The presence of microbiota greatly increased expression of mature $m i R-181 \mathrm{a}$ and $m i R-181 \mathrm{~b}$ in eWAT adipocytes (Fig. 4A-B). Notably, expression of $m i R-181 \mathrm{c}$ was primarily increased in eWAT stromal-vascular cells, but not adipocytes (fig. S7G), demonstrating that induction of $m i R-181$ family members by the microbiota is cell type-specific even within WAT. Of note, our previous results in DKO mice suggested that the miR-181c-d locus was not required for the observed phenotypes (Fig. 1 and 2). To further establish the role of the microbiota in regulating miR-181, we colonized GF mice with microbiota from SPF mice by co-housing and then measured expression of mature miR-181 species in eWAT adipocytes. Indeed, colonization of GF mice with the microbiota of SPF mice substantially increased eWAT adipocyte expression of miR-181a and miR-181b (Fig. 4C). Conversely, oral treatment of SPF mice with broad-spectrum antibiotics decreased miR-181 expression in eWAT in vivo (fig. S7H). Furthermore, HFD-induced up-regulation of adipocyte miR-181 expression during obesity was microbiota-dependent, as $m i R-181$ expression was abrogated in HFD-fed GF and antibiotic-treated mice (Fig. 4A-B, fig. S7I). Taken together, these results indicate that the microbiota regulates expression of the miR-181 family in eWAT adipocytes. Moreover, they suggest that a key mechanism by which the microbiota promotes IR and adiposity is through the regulation of this highly-conserved family of miRNAs in eWAT adipocytes. 


\section{Tryptophan-derived metabolites control miR-181 expression in WAT}

We then aimed to define the mechanisms by which the gut microbiota impacts miR-181 expression in adipocytes. The gut microbiota produces thousands of diffusible metabolites that regulate organs distal to the intestines (7). As LPS did not regulate miR-181 expression in vivo in SPF or GF mice (fig. S7E-F), we hypothesized that metabolites derived from these microbes might directly control miR-181 expression in WAT. More specifically, we postulated that $m i R-181$ expression is regulated by the relative abundance of microbiotaderived metabolites which induce or repress $m i R-181$ expression, and that disturbances in the relative abundances of these metabolites during DIO contribute to the development of obesity and IR. Previously well-characterized microbiota-derived metabolites reported to be immune or metabolic modulators, including short-chain fatty acids and bile acids, did not impact miR-181 expression in eWAT (fig. S8A-B). We therefore interrogated a previouslygenerated non-targeted metabolomic analysis of more than 600 metabolites in the cecal contents of HFD-fed mice (42). We reasoned that during DIO, the abundance of a metabolite acting as a positive regulator of $m i R-181$ should increase, whereas the abundance of a metabolite acting as a negative regulator of $m i R-181$ should decrease. The molecule most reduced in HFD-fed mice was the tryptophan-derived metabolite indole-3-carboxylic acid (I3CA) (Fig. 4D-E). Indole and its carboxylated form I3CA were produced by the microbiota from dietary tryptophan by the bacterial enzyme tryptophanase in the intestinal lumen (Fig. 4F). In agreement with our targeted metabolomic screening, metagenomic analysis revealed that the copy number of the tryptophanase (tnaA) gene within the gut microbiome was reduced in HFD-fed mice, as were the amounts of indole and indole derivatives in the feces and urine of these animals (fig. S8C-F). Bacteria-derived indole is absorbed by the host and metabolized in the liver to indoxyl sulfate, which is detectable in circulation (43). To determine whether the reduction of indole and indole-derived metabolites in the intestine during DIO led to a reduction in these metabolites in the peripheral circulation, we used targeted mass spectrometry to quantify the concentration of indoxyl sulfate in plasma from NCD- and HFD-fed WT mice. Indeed, indoxyl sulfate was absent in GF mice, reached approximately $1 \mathrm{mg} / \mathrm{ml}$ in the plasma of NCD-fed SPF mice, and was substantially reduced in HFD-fed SPF mice (Fig. 4G). We next assessed whether similar changes in indole or indole-derived metabolites were observed in a cohort of 38 obese and non-obese children at the Children's Hospital of Philadelphia. Despite the moderate number of participants, we found a reduction in indole in the blood of obese children (Fig. $4 \mathrm{H}$ ), consistent with our measurements in mouse plasma. Based on these observations, we hypothesized that indole or its derivatives such as indoxyl sulfate could be negative regulators of miR-181 expression in adipocytes and thereby mitigate the progression of obesity and IR.

To address this hypothesis, we used a well-characterized in vitro system of white adipocyte differentiation to determine the impact of tryptophan-derived metabolites on adipogenesis. Indole and indoxyl sulfate showed highly-specific and dose-dependent inhibition of adipocyte differentiation and lipid accumulation in vitro (Fig. 4I-J, fig. S8G-H). Both metabolites repressed $m i R-181 a$ and $m i R-181 b$ expression in vitro (Fig. $4 \mathrm{~K}$, fig. S8I). Moreover, overexpression of $m i R-181$ restored adipocyte differentiation and lipid accumulation in indole-treated cells (fig. S8J), suggesting that $m i R-181$ repression mediated 
some of the anti-adipogenic effects of indole in cultured white adipocytes. We aimed to establish whether restoring indole and indoxyl sulfate during HFD-feeding could repress $m i R-181$ expression in WAT and protect against DIO and IR. We administered indole in vivo and measured weight gain, glucose tolerance, and $m i R-181$ expression in eWAT adipocytes. We also validated the concentration of indoxyl sulfate in the circulation of treated mice by targeted mass spectrometry to ensure that the concentration of indole was equivalent to endogenous amounts present in NCD-fed mice (fig. S8K). Indeed, indole administration repressed white adipocyte $m i R-181$ expression and decreased the severity of DIO and glucose intolerance (Fig. 4L-O). However, indole administration did not protect against the progression of obesity and glucose intolerance in mice lacking miR-181 in adipocytes (Fig. 4P, fig. S8L). In concordance with our results showing that tryptophan-derived metabolites negatively regulated $m i R-181$ expression in WAT, we found that feeding mice a tryptophandeficient diet led to de-repression of miR-181 in white adipocytes in vivo (Fig. 4Q-R).

Altogether, these data suggest that de-repression of miR-181 expression in WAT contributes to the progression of obesity and IR due to reduced indole during DIO.

We aimed to determine whether colonizing mice with bacterial strains that do or do not produce indole could regulate weight gain and glucose homeostasis. We treated mice with broad-spectrum antibiotics, colonized them with a parental Escherichia coli (E. coli) strain expressing the tryptophanase gene tna $A$ or a derivative strain lacking this enzyme (tna $A$ $\mathrm{KO}$ ), and fed them a HFD (fig. S8M-P). In agreement with our metabolomic and indolesupplementation results, mice colonized with tna $A \mathrm{KO}$ strains had accelerated weight gain and decreased glucose tolerance relative to mice colonized with WT E. coli (fig. 4S-T, $\mathrm{S} 8 \mathrm{Q}$ ). Our data indicates that WAT $m i R-181$ expression is controlled by the relative abundance of microbiota-derived metabolites acting as positive and negative regulators of $m i R-181$ expression, and that indole and indole-derived metabolites including indoxyl sulfate are key negative regulators of $m i R-181$ expression in WAT. Furthermore, our results suggest that decreased amounts of indole metabolites in the context of nutritional surplus contribute to the progression of obesity and IR through their impact on WAT miR-181 expression. In summary, our results provide evidence of a miRNA family regulated by specific microbiota-derived metabolites that serves as a central regulator of WAT function. Moreover, this regulatory axis is critical for the development of obesity, IR and WAT inflammation (fig. S9).

\section{DISCUSSION}

Herein, we show that the gut microbiota specifically controlled expression of the miR-181 family in white adipocytes during homeostasis to regulate key pathways controlling adiposity, insulin sensitivity, and WAT inflammation in mice. Furthermore, HFD feeding altered the composition of the gut microbiota, resulting in decreases in microbiota-derived indole and its derivatives, leading to aberrant overexpression of $m i R$ - 181 in WAT adipocytes and the development of obesity, IR, and WAT inflammation. These results indicate that regulation of miR-181 specifically in white adipocytes serves as a key mechanism by which the microbiota tightly controls mammalian metabolism, independent of any role for miR-181 in other tissues. Moreover, our results suggest that changes in the composition of 
the microbiota triggered by environmental cues could contribute to the development of obesity-related disorders through dysregulation of $m i R-181$ in WAT.

Accumulating evidence suggests that many miRNAs evolved to act not as switches of specific, individual genes, but rather to modulate expression of large gene networks. Therefore, effects on single target genes might not explain the biological functions of an individual miRNA or miRNA family (9). In concordance with these observations, we showed that the $m i R-181$ family represses several critical genes in pathways controlling insulin signaling and WAT adipogenesis. These results suggest that regulation of $m i R-181$ serves as a rheostatic mechanism allowing rapid and global modulation of gene expression programs in white adipocytes in response to microbiota-derived signals. Furthermore, as miRNAs primarily control gene expression post-transcriptionally (44), this mechanism provides a potential means by which energy expenditure, adiposity, and insulin sensitivity could be tuned by environmental cues in a manner that does not require the more durable changes in gene expression triggered by transcriptional or epigenetic regulators.

Tryptophan is an essential amino acid that the microbiota can convert to indole compounds which accumulate to millimolar concentrations in the gut lumen and have the capacity to regulate gene expression in host intestinal cells and distal tissues $(43,45)$. It is therefore conceivable that control of $m i R-181$ expression in adipocytes evolved as a mechanism for monitoring nutritional intake, allowing the host to adjust anabolic pathways in response to nutrient availability, and that dysregulation of $m i R-181$ expression could lead to substantial alterations of metabolic processes with pathological consequences. Although indole and indoxyl sulfate repress miR-181 expression in adipocytes, our results in GF mice indicate that there must be positive signals necessary for $m i R-181$ expression in WAT at homeostasis and during DIO. Elucidating the nature of these signals will be crucial to understanding this critical pathway by which the microbiota regulates host metabolism and WAT inflammation.

There are several limitations to our study. We established that tryptophan-derived metabolites were reduced in the context of murine DIO in two separate vivariums and in an obese human pediatric cohort. However, future research is required to determine whether this phenomenon is generalizable under different environmental conditions such as additional animal vivariums, human cohorts, or upon administration of different obesogenic diets. Furthermore, our results using five different TcKO mouse strains coupled with the expression pattern of $m i R-181$ during DIO suggest that a large portion of the effects that this microRNA family exerts on host metabolism is due to its expression in WAT adipocytes. However, some of the effects that this microRNA family exerts on host metabolism are independent of its role in WAT. Identifying these WAT-independent mechanisms will be critical to fully elucidate the regulatory functions of $m i R-181$ and for the potential development of safe therapeutics targeting the $m i R-181$ family in the context of obesity. Future work will be needed to establish if the $m i R-181$ family is targetable in humans.

Altogether, our work proposes that regulation of miRNAs by microbiota-derived signals is a key mechanism controlling host metabolic processes and WAT inflammation, and that dysregulation of the expression of miRNAs as a consequence of changes in the abundance of microbial metabolites during gut microbiota dysbiosis contributes to the development of 
obesity and associated disorders. We also found that MIR-181 expression in WAT and the abundance of tryptophan-derived metabolites were dysregulated in obese humans. Therefore, our findings may have clinical implications, as they suggest that modulating MIR-181 expression by targeting specific microbiota-derived cues may be a potential therapeutic approach for the treatment of obesity and associated metabolic disorders.

\section{MATERIALS AND METHODS}

\section{Study design}

The overall objective of our study was to investigate the role of the $m i R-181$ family in regulating adipose tissue function and whole-body metabolism, and the mechanisms controlling miR-181 expression. To do so, we initially used a mouse model of DIO and compared the whole-body metabolic responses of WT and miR-181-deficient mice (DKO) during NCD and HFD feeding. As miR-181 was specifically up-regulated in WAT during DIO, we formally tested the cell type in which the miR-181 family mediates these metabolic phenotypes by generating five TcKO mice in which the miR-181 family was ablated: adipocytes, CD4+ T cells, Tregs, macrophages, and hepatocytes. To identify upstream signals regulating miR-181 in white adipocytes, we performed gene expression analyses on eWAT adipocytes from GF and SPF WT mice, cecal metabolomics and plasma mass spectrometry on HFD- and NCD-fed WT mice, as well as metabolite treatments of the 3T3L1A cell line. To address the role of the identified candidate metabolites in vivo, we administered indole to HFD-fed WT mice and miR-181-TcKO mice (Adipocytes, Fabp4 Cre). In addition, WT mice were fed a tryptophan-deficient diet, and antibiotic-treated WT mice colonized with tryptophanase-sufficient or tryptophanase-deficient strains of E. coli. Finally, to determine miR-181 expression and indole abundance during obesity in humans, we used human WAT biopsies and metabolomic analysis of serum, both with paired BMI values. Sources of mouse data included analysis of harvested tissues by qPCR, RNA-seq, flow cytometry, histology, and western blot, metabolic phenotyping of mice fed different diets or treated with specific compounds, metabolomic analysis of feces and serum, and experiments performed on mouse cell lines cultured in vitro. For in vivo metabolite studies, mice were randomized to control or treatment groups by cage. Where applicable, Cre recombinase-positive and -negative mice from the same litters were used as experimental and control groups, respectively. Our mouse studies were not blinded. Sample processing and statistical analysis were performed concurrently on experimental and control groups using identical methods. Numbers of replicates and statistical tests are indicated in figure legends.

\section{Pediatric cohort}

Obese (body mass index, BMI, >95th percentile for age, $n=19$ ) and non-obese (BMI <85th percentile for age, $n=19$ ) pediatric patients were recruited in accordance with an approved protocol from the Children's Hospital of Philadelphia Institutional Review Board. Each patient had BMI measured in a research setting, and had blood drawn from which serum was collected for non-targeted metabolomics. Adipose tissue samples were obtained from the University of Pennsylvania's Institute for Diabetes, Obesity, and Metabolism in accordance 
with the University of Pennsylvania Institutional Review Board. Total RNA was isolated from adipose tissue as described in the Supplement.

Mice

C57BL/6 (WT) mice and mice expressing Cre-recombinase (Albumin-Cre (B6.Cg-Speer6ps1Tg( $A l b$-cre) $21 \mathrm{Mgn} / \mathrm{J}), C d 4$ Cre (B6.Cg-Tg(Cd4-cre)1Cwi/BfluJ), Fabp4Cre (B6.Cg$\mathrm{Tg}$ (Fabp4-cre)1Rev/J), FoxP3-Cre (B6.129(Cg)-Foxp3tm4(YFP/icre)Ayr/J), LyzM-Cre (B6.129P2-Lyz2tm1(cre)Ifo/J)) were purchased from The Jackson Laboratory. Mice deficient in miR-181a1-b1, miR-181a2-b2, or miR-181c-d, or with the above loci flanked by loxP sites, were individually generated as previously described (12). miR-181a1-b1-/- and miR-181a2-b2-I- were then crossed to generate miR-181a1-b1; miR-181a2-b2-/- DKO mice. $m i R-181 a 1-b 1 f l / f l, m i R-181 a 2-b 2 f l / f l$, and $m i R-181 c-d f l / f l$ mice were crossed to generate miR-181a1-b1fl/fl; miR-181a2-b2fl/fl; miR-181c-dfl/fl mice which were then bred to mice expressing the Cre-recombinase under the control of the appropriate promoter as described above to generate TcKOs. Male mice were age-matched in all experiments, and cage-matched littermates were used for experiments when possible. All mice were bred and maintained under SPF conditions at an American Association for the Accreditation of Laboratory Animal Care accredited animal facility at the University of Pennsylvania. Mice were given ad libitum access to NCD or HFD and water. Mice were housed in accordance with the procedures outlined in the Guide for the Care and Use of Laboratory Animals under an animal study proposal approved by an Institutional Animal Care and Use Committee (IACUC). All mice used were between 3 and 22 weeks of age. GF C57BL/6 mice were maintained by the University of Pennsylvania Gnotobiotic Mouse Facility in accordance with all policies as listed above. Sample sizes are indicated in figure captions.

\section{Diet-induced obesity}

Unless otherwise specified, 6-week-old mice were either maintained on a normal chow diet (NCD) consisting of 5\% w/w fat (13\% kcal from fat) (Labdiet), or switched to a high-fat diet (HFD) consisting of 35\% w/w fat (60\% kcal from fat) (Research Diets) for specified periods of time before being sacrificed for experiments as described.

\section{Body composition analysis}

6-week-old male mice were fed either NCD (Labdiet) or HFD (Research Diets) for 12 weeks. At 18 weeks of age, mice were euthanized and NMR body composition analysis performed with a LF50 minispec from Bruker.

\section{Epididymal white adipose tissue (eWAT) histology}

6-week-old male mice were fed either NCD or HFD for 12 weeks. At 18 weeks of age mice were euthanized, epididymal fat pads were excised, washed in PBS, blotted dry, and weighed. Fat pads were then fixed for 48 hours in $4 \%$ paraformaldehyde (Sigma) and delivered to the University of Pennsylvania Histology Core for paraffin embedding, sectioning, and hematoxylin and eosin (H\&E) staining. Slides were imaged and adipocyte cell sizes within a 20x magnification field were determined using Image $\mathrm{J}$. 


\section{Fasted plasma insulin}

WT and DKO mice were fed either NCD or HFD for 12 weeks. Blood was collected following a 16-hour overnight fast and plasma was isolated by centrifugation. Samples were then sent to Vanderbilt University Metabolic Core supported by NIH grants DK059637 and DK020593 for analysis. Homeostatic Model Assessment of Insulin Resistance (HOMA-IR) values were calculated using fasted glucose and plasma values in the following equation: HOMA-IR $=($ Glucose $x$ Insulin $) / 405$.

\section{Thermoneutral metabolic cage analysis and cold challenge}

Analysis of mouse whole-body metabolism was performed by the Penn Diabetes Mouse Phenotyping, Physiology and Metabolism Core, which is supported by Penn Diabetes Research Center grant P30-DK19525. For thermoneutral metabolic cage analysis, WT and DKO mice fed either NCD or HFD were maintained in a Comprehensive Laboratory Animal Monitoring System (CLAMS) at $24^{\circ} \mathrm{C}$ for 48 (HFD, diet started at 8-10 weeks and maintained for 6 weeks) or 72 (NCD) hours on a standard 12:12 light-dark cycle with ad libitum access to food and water. For the cold exposure stress test, WT and DKO mice fed NCD or HFD were maintained in CLAMS at $4^{\circ} \mathrm{C}$ for 7 (HFD diet started at 8-10 weeks and maintained for 6 weeks) or 25 (NCD) hours with free access to food and water. Following cold exposure, mice were immediately sacrificed, and tissues were processed for downstream analysis as described in the Supplement. For $\mathrm{VO}_{2}, \mathrm{VCO}_{2}$, calculated heat production, movement, and food consumption, data collected in the first hour of the cold stress test and the first 24 hours of the thermoneutral metabolic cage experiment were discarded prior to data analysis. For $\mathrm{VO}_{2}, \mathrm{VCO}_{2}$, and calculated heat production, measurements were normalized to lean mass of individual mice.

\section{In vivo LPS treatment}

First, WT male mice at 8 weeks of age housed in SPF conditions and fed NCD were injected intraperitoneally with LPS at a dose of $1 \mu \mathrm{g} / \mathrm{g}$. 4 hours later, mice were euthanized by $\mathrm{CO}_{2}$ inhalation and tissue was harvested for analysis of miR-181 expression. Second, WT male mice at 10-12 weeks of age housed in germ-free (GF) conditions and fed NCD were treated with $50 \mu \mathrm{g} / \mathrm{mL}$ LPS in drinking water for 2 weeks. Mice were then euthanized by $\mathrm{CO}_{2}$ inhalation and tissue harvested for quantification of $m i R-181$ expression.

\section{Antibiotic treatment}

To deplete the gastrointestinal microbiota, mice were treated with a combination of ampicillin, vancomycin, metronidazole, and kanamycin at a concentration of 1 gram each per liter of drinking water. After 2 weeks of treatment, the concentration of vancomycin was reduced to $0.5 \mathrm{~g} / \mathrm{L}$ for the indicated durations.

\section{In vivo microbiota-derived metabolite treatment}

WT male mice at 6-8 weeks of age were housed in SPF conditions and fed a NCD. Sterile drinking water was supplemented with select microbiota-derived metabolites at previously characterized concentrations. After 6 weeks of treatment, mice were euthanized by $\mathrm{CO}_{2}$ inhalation and tissues were harvested for quantification of $m i R-181$ expression. All reagents 
were purchased from Sigma. The concentrations of metabolites administered in drinking water were as follows: short chain fatty-acids, $100 \mathrm{mM}$ (acetate: $0.82 \mathrm{~g} / 100 \mathrm{~mL} \mathrm{H}_{2} \mathrm{O}$; propionate: $0.96 \mathrm{~g} / 100 \mathrm{~mL}$; butyrate: $\left.1.1 \mathrm{~g} / 100 \mathrm{~mL} \mathrm{H}_{2} \mathrm{O}\right)$; TMAO, $50 \mu \mathrm{M}\left(1 \mathrm{~g} / 100 \mathrm{~mL} \mathrm{H}_{2} \mathrm{O}\right)$; bile acid pool (deoxycholic acid and taurocholic acid), $0.7 \mu \mathrm{M}$ deoxycholic acid, $0.6 \mu \mathrm{M}$ taurocholic acid $\left(300 \mathrm{mg} / 100 \mathrm{~mL} \mathrm{H}_{2} \mathrm{O}\right)$; branched-chain amino acid pool (leucine, isoleucine, and valine), $15 \mu \mathrm{M}$ each $\left(1 \mathrm{~g} / 100 \mathrm{~mL} \mathrm{H}_{2} \mathrm{O}\right)$.

\section{In vivo tryptophan-derived metabolite studies}

3-week-old male C57BL/6J mice were obtained from The Jackson Laboratory, received a bedding exchange, and were allowed to acclimate to our mouse facility for 1 week. Mice were then intraperitoneally injected with $100 \mathrm{uL}$ of indole at $50 \mathrm{mg} / \mathrm{kg}$ diluted in solvent (4mL polyethylene glycol, $10 \mu \mathrm{L}$ benzyl alcohol, $1 \mathrm{~mL}$ ethanol, and $4.95 \mathrm{~mL}$ nuclease free water) or solvent alone 3 times per week. After 1 week of injections, mice were fed HFD ad libitum with free access to water. Mice were injected 3 times per week for 8 weeks and weights were measured post-injection. Mice were sacrificed at 13 weeks of age for tissue collection and miR-181 expression analysis. For experiments using miR-181a1b1/a2b2/cd fl/fl; Fabp4-Cre positive mice (triple conditional knockout, TcKO), 10-week-old mice were fed HFD starting 5 days prior to indole treatment. TcKO mice were then weighed and intraperitoneally injected with $100 \mathrm{uL}$ of indole at $50 \mathrm{mg} / \mathrm{kg}$ diluted in solvent or solvent alone 3 times per week.

\section{Glucose tolerance test (GTT) and insulin tolerance test (ITT)}

GTTs and ITTs were performed on NCD- or HFD-fed mice at 18 weeks of age. Following an overnight fast of 16 hours, the tail vein was nicked and baseline blood glucose concentrations were determined with a Contour glucometer. Each mouse then received a $1 \mathrm{~g} / \mathrm{kg}$ intraperitoneal (i.p.) injection of $10 \%$ D-Glucose (Sigma) (GTT) or 0.75 units $/ \mathrm{kg}$ i.p injection of human insulin (Sigma) (ITT). Blood glucose concentrations were then recorded.

\section{Targeted mass spectrometry in mouse plasma}

The concentrations of indoxyl sulfate (MW: 213) and indole (MW: 117) in plasma were detected using an API 5000 LC/MS/MS system in ESI negative or ESI positive ionization mode, respectively. A product mass of 132 and 89.2 for indoxyl sulfate and indole, respectively, were selected for analysis. Additionally, an internal standard curve of fexofenadine was utilized for both molecules. For chromatography, an Xbridge C18 column was used with a particle size of $3.5 \mathrm{um}$ and a dimension of $2.1 \times 20 \mathrm{~mm}$ for both molecules. Indoxyl sulfate utilized $10 \mathrm{mM}$ ammonium acetate in water at a $\mathrm{pH}$ of 6.5 interchanged with acetonitrile, while indole required a mobile phase of $0.1 \%$ formic acid in water interchanged with acetonitrile. Washes of 80:20 acetonitrile:water and 45:45:10 acetonitrile:isopropyl alcohol:acetone where used between samples for both indoxyl sulfate and indole.

\section{Colonization of mice with $E$. coli sufficient and deficient for the tryptophanase gene}

Briefly, following a week-long facility acclimation and bedding exchange, 6-week-old SPF C57BL/6J male mice were given antibiotic water containing $1 \mathrm{~g} / \mathrm{L}$ of ampicillin, vancomycin, metronidazole, and kanamycin for 6 days. On day 6 mice were taken off 
antibiotic water and given ad libitum access to facility water. The following day, mice were orally gavaged with $100 \mu \mathrm{L}$ of PBS or with $1-2.0 \times 10^{8} \mathrm{CFU}$ of one of the following $E$. coli strains: WT parent strain BW25113 (OEC5042) or one of two JW3686 strains with independent tryptophanase (tnaA) deletions (OEC4987-213606712 or OEC4987200828669). Keio Collection strains were purchased from Dharmacon, Inc. Bacteria was cultured overnight in LB (WT) or low salt LB with kanamycin (tnaA KO), washed with $1 \mathrm{~mL}$ PBS, centrifuged at $3900 \mathrm{rpm}$ for 15 minutes, then resuspended in $1 \mathrm{~mL}$ PBS. Mice were colonized and weighed 3 times per week for 2 weeks then were fed HFD continuing on the same schedule of weighing and colonization. A bedding exchange with facility mice was performed 3 times per week for mice gavaged with PBS.

Colonization was confirmed by performing both endpoint and qPCR. Briefly, feces was collected from individual mice and genomic DNA was extracted using the QIAamp PowerFecal Pro DNA Kit (Qiagen) followed by endpoint PCR using the TopTaq Master Mix kit (Qiagen). The tnaA upstream ACATCCTTATAGCCACTCTGTAGTATTAATTAAACTTCTTTAAGTTTTGC and tna $A$ downstream -

ACATCCTTATAGCCACTCTGTAGTATTAATTAAACTTCTTTAAGTTTTGC primers were used to confirm the presence of Keio Collection E. coli strains via endpoint PCR. The tna $A$ knockout $E$. coli strains (JW3686) were amplified using the tha $A$ downstream and k1 CAGTCATAGCCGAATAGCCT primers. Real-Time PCR was performed using the extracted genomic DNA and the KAPA SYBR FAST qPCR Master Mix (2X) Kit (KAPA Biosystems). Quantitative PCR was performed to estimate relative bacterial abundance of $E$. coli (uidA forward - ATGGAATTTCGCCGATTTTGC, uidA reverse ATTGTTTGCCTCCCTGCTGC) and JW3686 knockouts (tnaA downstream and K1 primers) all normalized to total bacterial load using 16s rRNA primers (27 forward AGAGTTTGATCCTGGCTCAG, 1525 reverse - AAGGAGGTGWTCCARCC).

\section{Quantification and statistical analysis}

Statistical analyses were performed in Prism 7. Data were tested for normality using either a Kolmogorov-Smirnov test or a Shapiro-Wilk test and analysis performed using two-tailed Student's t-test or one-way or two-way ANOVA with Tukey's post hoc for parametric data. For non-parametric data, a Mann-Whitney test or a Kruskal-Wallis with Dunn's multiple comparisons test was performed as indicated in the figure legends. In all cases $\mathrm{p} \leq 0.05$ was considered statistically significant. All error bars represent SEM.

\section{Supplementary Material}

Refer to Web version on PubMed Central for supplementary material.

\section{ACKNOWLEDGEMENTS}

We thank the University of Pennsylvania Diabetes Research Center (DRC) and Dr. Joseph Baur for the use of the Mouse Phenotyping, Physiology and Metabolism Core. We would like to thank Dr. Michael Tordoff of the Monell Chemical Senses Center for providing access to their Buker LF50 minispec. We would also like to thank the DRC Metabolomics Core.

FUNDING 
The work in this manuscript was supported by funds from CHOP, UPenn IFI and IDOM pilot projects, NIH R21AI128060, R21DK111755, and R01HL136572, the PEW Biomedical Scholars award, and the Burroughs Wellcome Fund investigator in the pathogenesis of infectious diseases award (J.H-M.); M.T.J. by NIH T32AI070077-09; W.K.M. by NIH T32AI055428 and F31AI124538; J.J.K. by NIH T32DK007780 and F30HL138739; S.P.S. by NIH F30DK094708; E.J.W. by AI105343, AI108545, AI117950, AI082630, and CA210944; E.J.W. is a member of the Parker Institute for Cancer Immunotherapy which supported the UPenn cancer immunotherapy program; SEH by NIH 5K12HD043245 and T32-HD043021, Pennsylvania Allergy Education Research Fund and the University of Pennsylvania Diabetes Research Center (Pilot Grant).

\section{REFERENCES}

1. Gesta S, Tseng YH, Kahn CR, Developmental origin of fat: tracking obesity to its source. Cell 131, 242-256 (2007). [PubMed: 17956727]

2. Hajer GR, van Haeften TW, Visseren FL, Adipose tissue dysfunction in obesity, diabetes, and vascular diseases. European heart journal 29, 2959-2971 (2008). [PubMed: 18775919]

3. Suarez-Zamorano N. et al., Microbiota depletion promotes browning of white adipose tissue and reduces obesity. Nat Med 21, 1497-1501 (2015). [PubMed: 26569380]

4. Backhed F. et al., The gut microbiota as an environmental factor that regulates fat storage. Proc Natl Acad Sci U S A 101, 15718-15723 (2004). [PubMed: 15505215]

5. Backhed F, Manchester JK, Semenkovich CF, Gordon JI, Mechanisms underlying the resistance to diet-induced obesity in germ-free mice. Proc Natl Acad Sci U S A 104, 979-984 (2007). [PubMed: 17210919]

6. Khan MT, Nieuwdorp M, Backhed F, Microbial modulation of insulin sensitivity. Cell metabolism 20, 753-760 (2014). [PubMed: 25176147]

7. Martin FP et al., A top-down systems biology view of microbiome-mammalian metabolic interactions in a mouse model. Molecular systems biology 3, 112 (2007). [PubMed: 17515922]

8. Wang Z. et al., Gut flora metabolism of phosphatidylcholine promotes cardiovascular disease. Nature 472, 57-63 (2011). [PubMed: 21475195]

9. Bartel DP, Chen CZ, Micromanagers of gene expression: the potentially widespread influence of metazoan microRNAs. Nat Rev Genet 5, 396-400 (2004). [PubMed: 15143321]

10. Davalos A. et al., miR-33a/b contribute to the regulation of fatty acid metabolism and insulin signaling. Proc Natl Acad Sci U S A 108, 9232-9237 (2011). [PubMed: 21576456]

11. Trajkovski M. et al., MicroRNAs 103 and 107 regulate insulin sensitivity. Nature 474, 649-653 (2011). [PubMed: 21654750]

12. Williams A, Henao-Mejia J, Harman CC, Flavell RA, miR-181 and metabolic regulation in the immune system. Cold Spring Harb Symp Quant Biol 78, 223-230 (2013). [PubMed: 24163395]

13. Henao-Mejia J. et al., The microRNA miR-181 is a critical cellular metabolic rheostat essential for NKT cell ontogenesis and lymphocyte development and homeostasis. Immunity 38, 984-997 (2013). [PubMed: 23623381]

14. Romieu I. et al., Energy intake and other determinants of relative weight. Am J Clin Nutr 47, 406412 (1988). [PubMed: 3348153]

15. Tschop MH et al., A guide to analysis of mouse energy metabolism. Nature methods 9, 57-63 (2011). [PubMed: 22205519]

16. Bluher M. et al., Adipose tissue selective insulin receptor knockout protects against obesity and obesity-related glucose intolerance. Dev Cell 3, 25-38 (2002). [PubMed: 12110165]

17. Hotamisligil GS, Inflammation and metabolic disorders. Nature 444, 860-867 (2006). [PubMed: 17167474]

18. Weisberg SP et al., Obesity is associated with macrophage accumulation in adipose tissue. The Journal of clinical investigation 112, 1796-1808 (2003). [PubMed: 14679176]

19. Molofsky $\mathrm{AB}$ et al., Innate lymphoid type 2 cells sustain visceral adipose tissue eosinophils and alternatively activated macrophages. J Exp Med 210, 535-549 (2013). [PubMed: 23420878]

20. Winer S. et al., Normalization of obesity-associated insulin resistance through immunotherapy. Nat Med 15, 921-929 (2009). [PubMed: 19633657] 
21. Olefsky JM, Glass CK, Macrophages, inflammation, and insulin resistance. Annu Rev Physiol 72, 219-246 (2010). [PubMed: 20148674]

22. Lumeng CN, Bodzin JL, Saltiel AR, Obesity induces a phenotypic switch in adipose tissue macrophage polarization. The Journal of clinical investigation 117, 175-184 (2007). [PubMed: 17200717]

23. Wu D. et al., Eosinophils sustain adipose alternatively activated macrophages associated with glucose homeostasis. Science (New York, N.Y.) 332, 243-247 (2011).

24. Ilan Y. et al., Induction of regulatory T cells decreases adipose inflammation and alleviates insulin resistance in ob/ob mice. Proc Natl Acad Sci U S A 107, 9765-9770 (2010). [PubMed: 20445103]

25. Basse AL et al., Global gene expression profiling of brown to white adipose tissue transformation in sheep reveals novel transcriptional components linked to adipose remodeling. BMC genomics 16, 215 (2015). [PubMed: 25887780]

26. Boender AJ, van Gestel MA, Garner KM, Luijendijk MC, Adan RA, The obesity-associated gene Negr1 regulates aspects of energy balance in rat hypothalamic areas. Physiological reports 2, (2014).

27. Forzati F. et al., CBX7 gene expression plays a negative role in adipocyte cell growth and differentiation. Biology open 3, 871-879 (2014). [PubMed: 25190058]

28. Heine PA, Taylor JA, Iwamoto GA, Lubahn DB, Cooke PS, Increased adipose tissue in male and female estrogen receptor-alpha knockout mice. Proc Natl Acad Sci U S A 97, 12729-12734 (2000). [PubMed: 11070086]

29. Mukherji A, Kobiita A, Chambon P, Shifting the feeding of mice to the rest phase creates metabolic alterations, which, on their own, shift the peripheral circadian clocks by 12 hours. Proc Natl Acad Sci U S A 112, E6683-6690 (2015). [PubMed: 26627259]

30. Withers DJ et al., Disruption of IRS-2 causes type 2 diabetes in mice. Nature 391, 900-904 (1998). [PubMed: 9495343]

31. Mavilio M. et al., A Role for Timp3 in Microbiota-Driven Hepatic Steatosis and Metabolic Dysfunction. Cell reports 16, 2269 (2016). [PubMed: 27558826]

32. Podrini C. et al., MacroH2A1 isoforms are associated with epigenetic markers for activation of lipogenic genes in fat-induced steatosis. Faseb j 29, 1676-1687 (2015). [PubMed: 25526730]

33. Gray S. et al., Regulation of gluconeogenesis by Kruppel-like factor 15. Cell metabolism 5, 305312 (2007). [PubMed: 17403374]

34. Crutzen R. et al., Anoctamin 1 (Ano1) is required for glucose-induced membrane potential oscillations and insulin secretion by murine beta-cells. Pflugers Archiv : European journal of physiology 468, 573-591 (2016). [PubMed: 26582426]

35. Iguchi H. et al., SOX6 attenuates glucose-stimulated insulin secretion by repressing PDX1 transcriptional activity and is down-regulated in hyperinsulinemic obese mice. The Journal of biological chemistry 280, 37669-37680 (2005). [PubMed: 16148004]

36. Tilg H, Moschen AR, Adipocytokines: mediators linking adipose tissue, inflammation and immunity. Nat Rev Immunol 6, 772-783 (2006). [PubMed: 16998510]

37. Ter Horst KW et al., Methods for quantifying adipose tissue insulin resistance in overweight/obese humans. Int J Obes (Lond) 41, 1288-1294 (2017). [PubMed: 28465607]

38. Orr JS, Kennedy AJ, Hasty AH, Isolation of adipose tissue immune cells. J Vis Exp, e50707 (2013). [PubMed: 23728515]

39. Grant RW, Dixit VD, Adipose tissue as an immunological organ. Obesity 23, 512-518 (2015). [PubMed: 25612251]

40. Brestoff Jonathan R., D. Artis, Immune Regulation of Metabolic Homeostasis in Health and Disease. Cell 161, 146-160 (2015). [PubMed: 25815992]

41. Caricilli AM, Saad MJ, The role of gut microbiota on insulin resistance. Nutrients 5, 829-851 (2013). [PubMed: 23482058]

42. Thaiss CA et al., Persistent microbiome alterations modulate the rate of post-dieting weight regain. Nature 540, 544-551 (2016). [PubMed: 27906159]

43. Wikoff WR et al., Metabolomics analysis reveals large effects of gut microflora on mammalian blood metabolites. Proc Natl Acad Sci U S A 106, 3698-3703 (2009). [PubMed: 19234110] 
44. Lai EC, Micro RNAs are complementary to 3' UTR sequence motifs that mediate negative posttranscriptional regulation. Nat Genet 30, 363-364 (2002). [PubMed: 11896390]

45. Krishnan S. et al., Gut Microbiota-Derived Tryptophan Metabolites Modulate Inflammatory Response in Hepatocytes and Macrophages. Cell reports 23, 1099-1111 (2018). [PubMed: 29694888]

46. Marco-Sola S, Sammeth M, Guigo R, Ribeca P, The GEM mapper: fast, accurate and versatile alignment by filtration. Nature methods 9, 1185-1188 (2012). [PubMed: 23103880]

47. Lu W. et al., Metabolomic analysis via reversed-phase ion-pairing liquid chromatography coupled to a stand alone orbitrap mass spectrometer. Analytical chemistry 82, 3212-3221 (2010). [PubMed: 20349993]

48. Papazyan R. et al., Physiological Suppression of Lipotoxic Liver Damage by Complementary Actions of HDAC3 and SCAP/SREBP. Cell metabolism 24, 863-874 (2016). [PubMed: 27866836]

49. Melamud E, Vastag L, Rabinowitz JD, Metabolomic analysis and visualization engine for LC-MS data. Analytical chemistry 82, 9818-9826 (2010). [PubMed: 21049934]

50. Skinner AC, Skelton JA, Prevalence and trends in obesity and severe obesity among children in the United States, 1999-2012. JAMA pediatrics 168, 561-566 (2014). [PubMed: 24710576] 
A

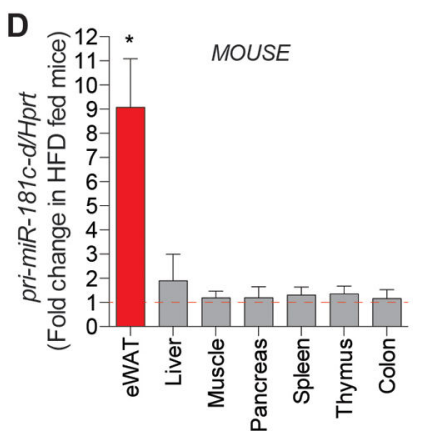

B

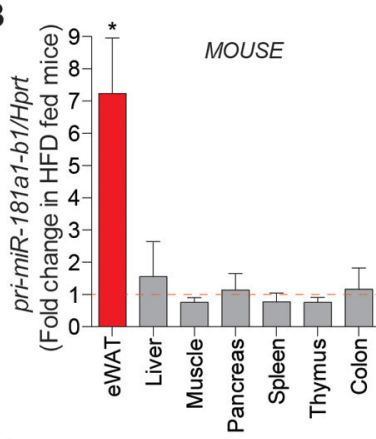

E

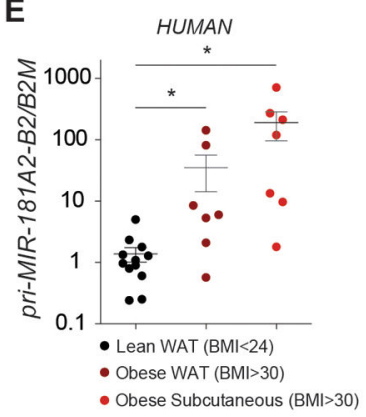

C

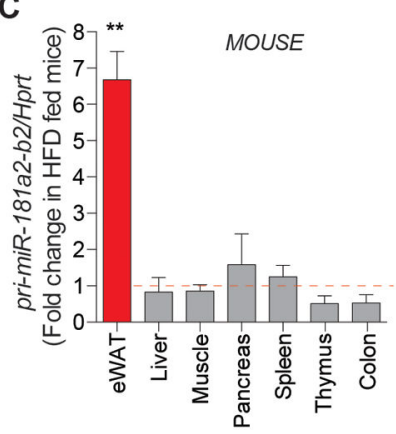

$F$

$\mathrm{F}_{50} \cdot-$ WTNCD

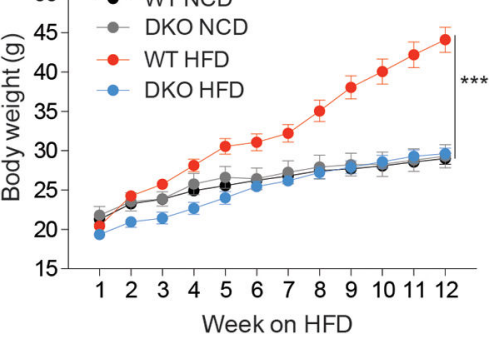

G

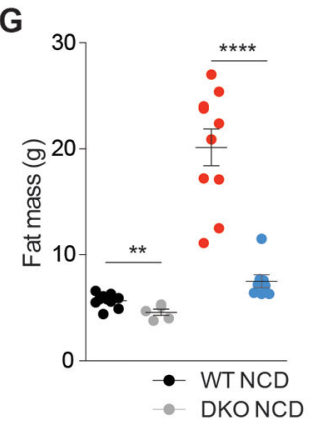

I

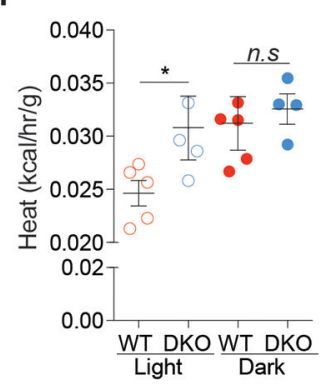

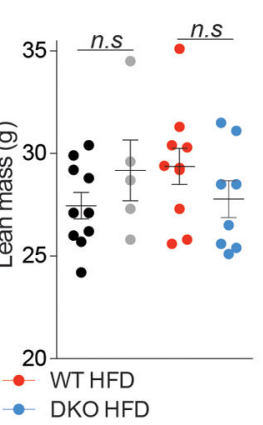

$\mathrm{J}$

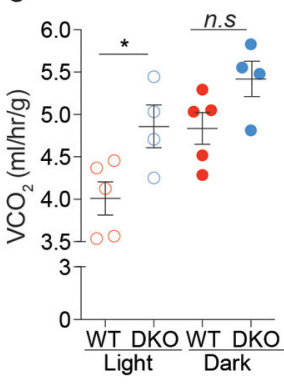

H EWATHFD-fed
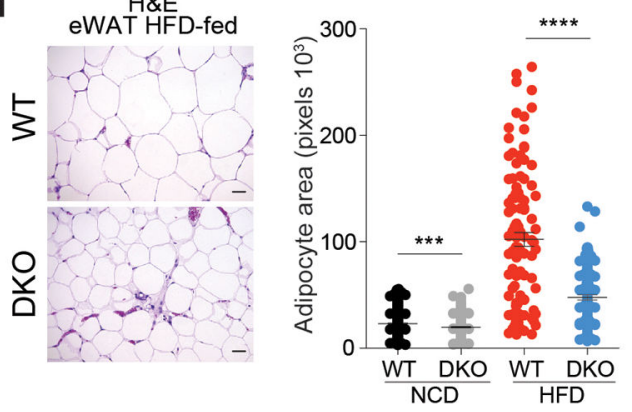

$\mathbf{L}$

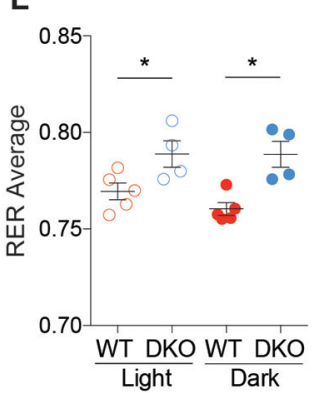

Figure 1. The miR-181 family is a critical regulator of adipose tissue function.

(A) Genomic location of the three miR-181 clusters (miR-181a1-b1, miR-181a2-b2, and $m i R-181 c-d$ ) in mice. (B-D) Expression of pri-miR-181 clusters relative to Hprt in tissue from mice fed a normocaloric diet (NCD) or a high-fat diet (HFD), shown as fold change relative to NCD-fed values ( $\mathrm{n}=3$ per group, two independent experiments). (E) Human priMIR-181A2-B2 expression in lean subcutaneous (BMI $<24 ; \mathrm{n}=12)$, obese subcutaneous (BMI $>30 ; n=7)$, and obese visceral WAT (BMI $>30 ; n=7)$. Each dot represents a single

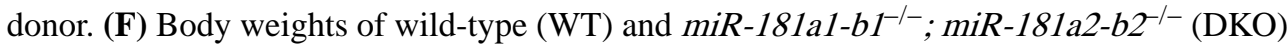


mice fed a NCD or HFD from 6 to 18 weeks of age (WT NCD n=10, DKO NCD n=5, WT HFD n=10, DKO HFD n=8). (G) Body composition by magnetic resonance imaging (MRI) of WT and DKO mice fed a NCD or HFD from 6 to 18 weeks of age (one dot per mouse; WT NCD n=10, DKO NCD n=5, WT HFD n=10, DKO HFD n=8). (H) Representative hematoxylin and eosin (H\&E) images of epididymal white adipose tissue (eWAT) from WT and DKO mice after 12 weeks of HFD; scale bar, $50 \mu \mathrm{m}$. Quantification of individual adipocytes within a 20x field (one dot per cell; WT NCD n=5, DKO NCD n=4, WT HFD $\mathrm{n}=5$, DKO HFD n=3). (I-L) Evaluation of whole-body metabolism by Comprehensive Laboratory Animal Monitoring System (CLAMS) of WT $(n=5)$ and DKO $(n=4)$ mice fed a HFD for 6 weeks starting at 8-10 weeks of age. (I) Average calculated heat production, (J) rate of $\mathrm{CO}_{2}$ elimination, $(\mathbf{K})$ rate of $\mathrm{O}_{2}$ consumption, and $(\mathbf{L})$ calculated respiratory exchange ratio (RER). One dot per mouse. Error bars indicate mean \pm SEM. Two-tailed Student's $t$ test $(\mathbf{B}, \mathbf{D}, \mathbf{I}, \mathbf{J}, \mathbf{K}, \mathbf{L})$, one-way ANOVA (E), two-way ANOVA (F), or MannWhitney test $(\mathbf{C}, \mathbf{G}, \mathbf{H}),{ }^{*} \mathrm{p}<0.05,{ }^{* *} \mathrm{p}<0.01$, and ${ }^{* *} \mathrm{p}<0.001$. 

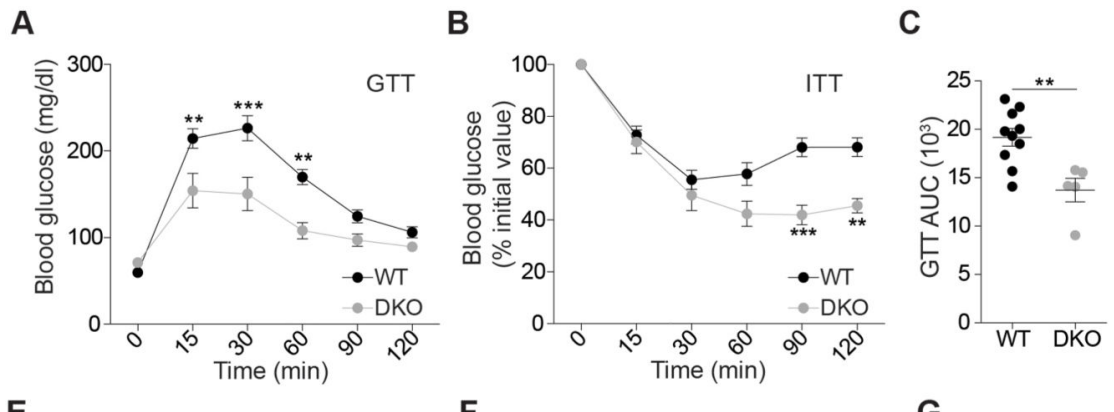

D
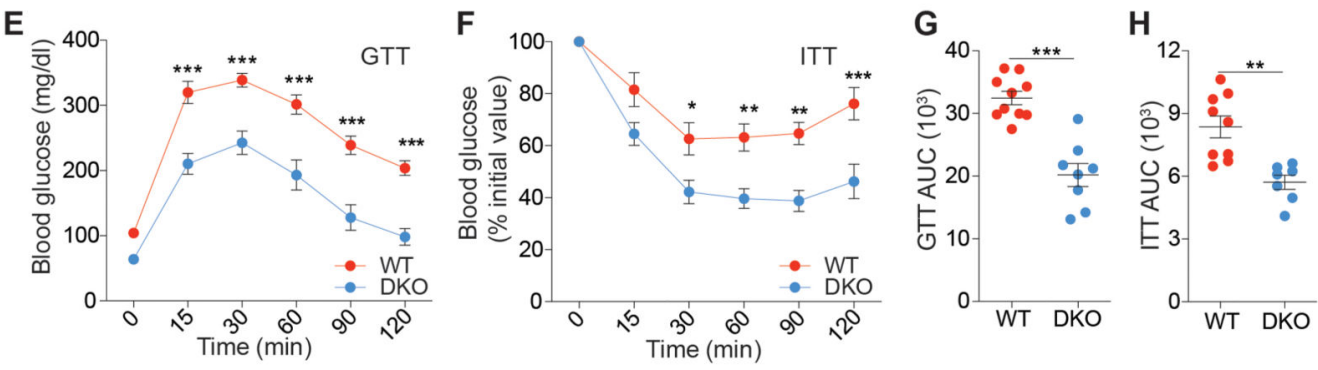

I

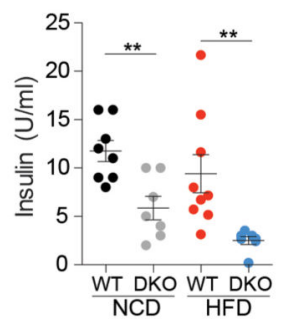

L
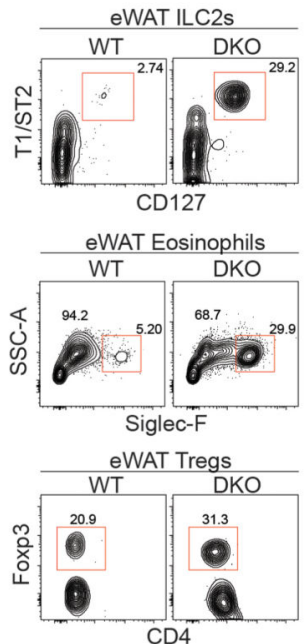

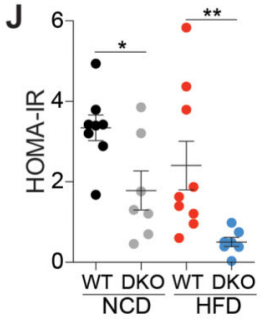

M
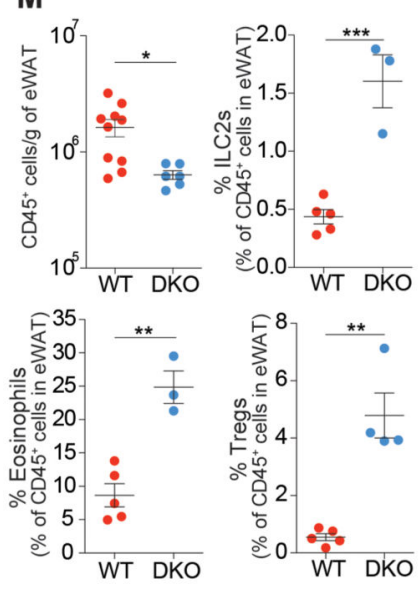

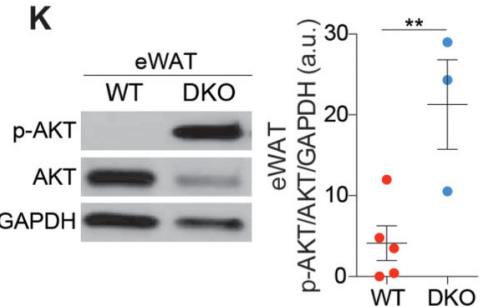

$\mathbf{N}$

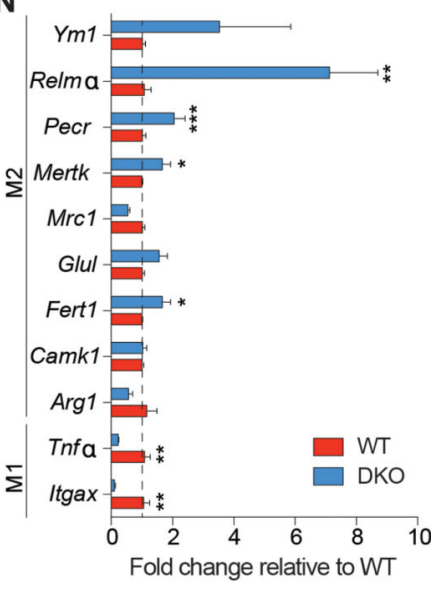

Figure 2. The miR-181 family regulates insulin sensitivity and promotes a pro-inflammatory status in WAT during obesity.

(A) Glucose tolerance test (GTT) of wild-type (WT) and miR-181a1-b1-1-; miR-181a2$b 2^{-1-}$ (DKO) mice fed a NCD. (B) Insulin tolerance test (ITT) of WT and DKO mice fed a NCD. (C) GTT area under the curve (AUC) values for WT $(n=10)$ and DKO $(n=5)$ NCD mice. (D) ITT AUC values for WT $(n=10)$ and DKO $(n=4)$ NCD mice. (E) GTT of WT and DKO mice fed a HFD from age 6 to 18 weeks. (F) ITT of WT and DKO mice fed a HFD at age 6 to 18 weeks. (G) GTT AUC values for WT $(n=10)$ and DKO $(n=8)$ HFD mice. (H) 
ITT AUC values for WT ( $\mathrm{n}=9$ ) and DKO ( $\mathrm{n}=7)$ HFD mice. One dot per mouse. (I) Serum insulin and (J) homeostatic model assessment-insulin resistance (HOMA-IR) index values for WT and DKO mice fed a NCD or HFD for 12 weeks. (One dot per mouse; WT NCD $\mathrm{n}=8$, DKO NCD n=7, WT HFD n=9 and DKO HFD n=7). (K) Representative western blot for $\mathrm{p}$-AKT, AKT, and GAPDH protein in eWAT of WT $(n=5)$ and DKO $(n=3)$ mice fed a HFD from 6 to 18 weeks of age. Mice were fasted for 4 hours prior to i.p. insulin injection and after 15 minutes, eWAT was snap frozen. p-AKT expression is shown normalized by total AKT and GAPDH. One dot per mouse per lane. a.u. = arbitrary unit. $(\mathbf{L})$

Representative flow cytometry plots of group 2 innate lymphoid cells (ILC2), eosinophils, and T-regulatory cells (Tregs) isolated from eWAT of WT and DKO mice fed a HFD from 6 to 18 weeks of age. (M) Quantification of $(\mathbf{L})$ total lymphocytes per gram of adipose tissue (AT) and percentage of indicated cell types within the CD45+ immune cell population. (One dot per mouse; WT HFD n=5-10, DKO HFD n=3-6). (N) Expression of M1 and M2 genes in eWAT of HFD-fed mice (WT n=5, DKO n=4) normalized to Hprt and shown as fold change relative to WT. Error bars show mean \pm SEM. Two-tailed Student's $t$-test $(\mathbf{D}, \mathbf{G}, \mathbf{H}$, $\mathbf{K}, \mathbf{N})$, two-way ANOVA (A, B, E, F), or Mann-Whitney test $(\mathbf{C}, \mathbf{I}, \mathbf{J}, \mathbf{M}),{ }^{*} \mathrm{p}<0.05,{ }^{* *} \mathrm{p}<$ $0.01,{ }^{* * * *} \mathrm{p}<0.001$. 

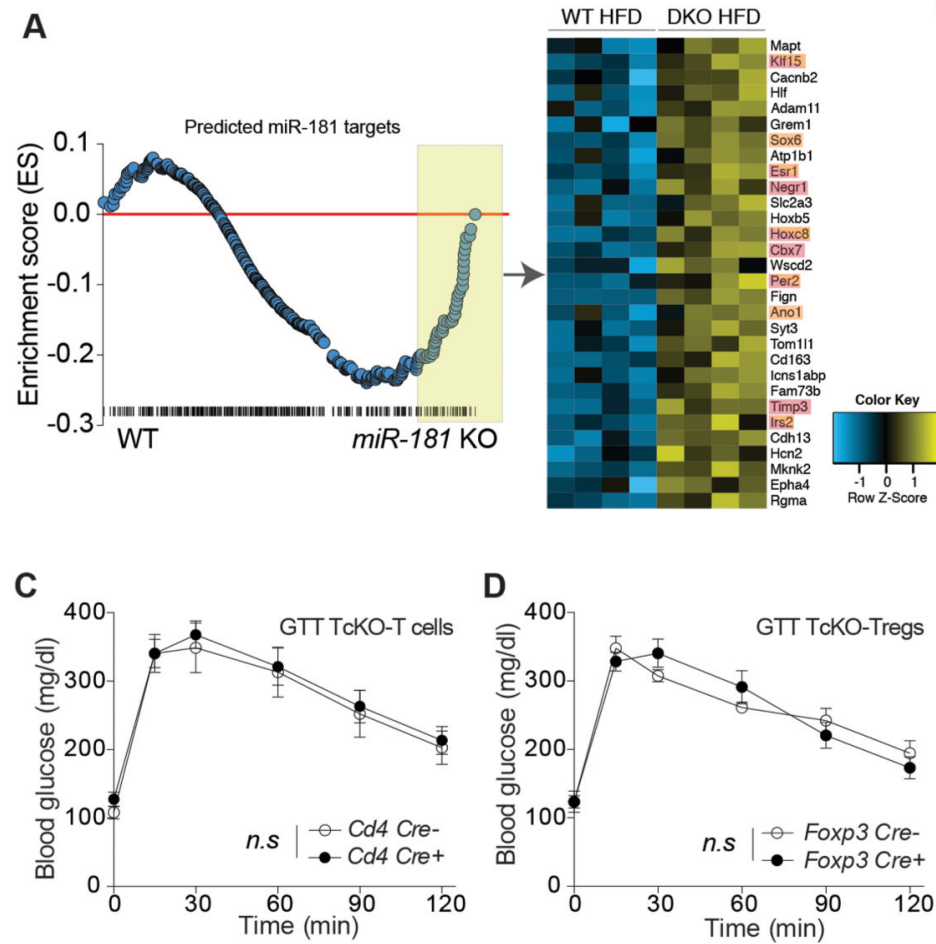

F
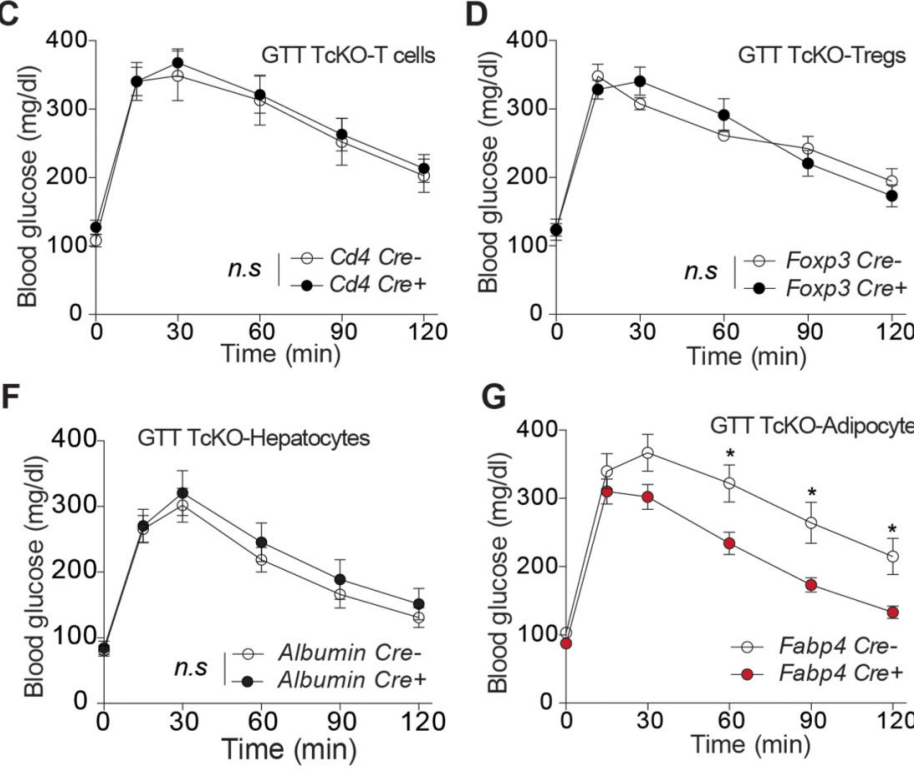

G
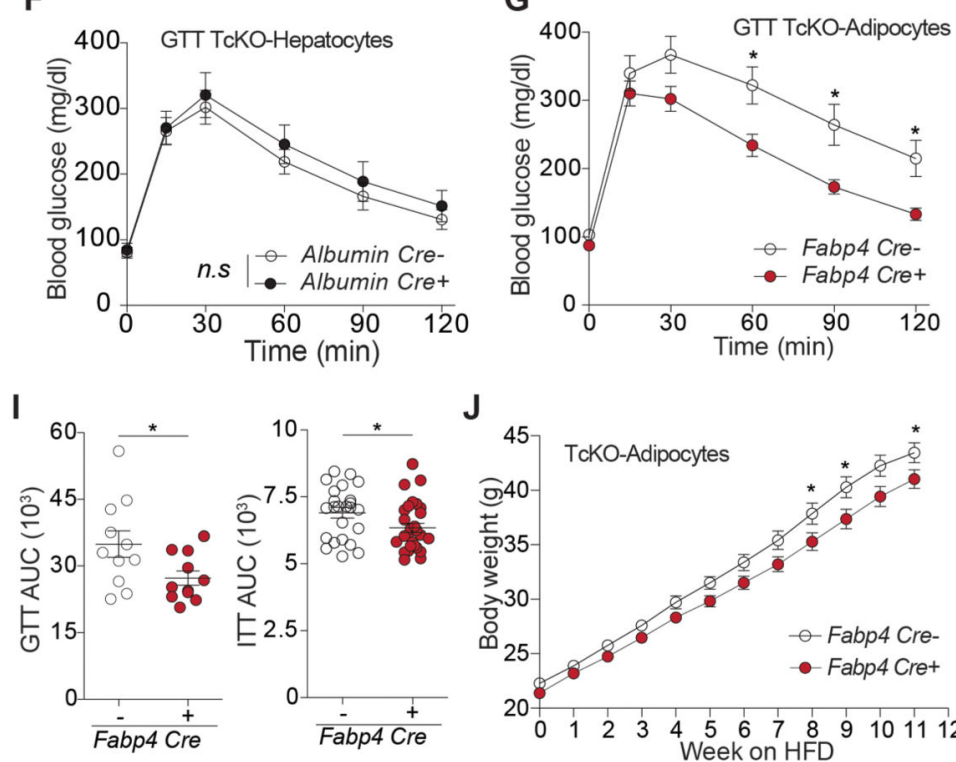

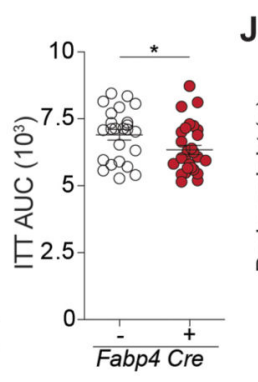

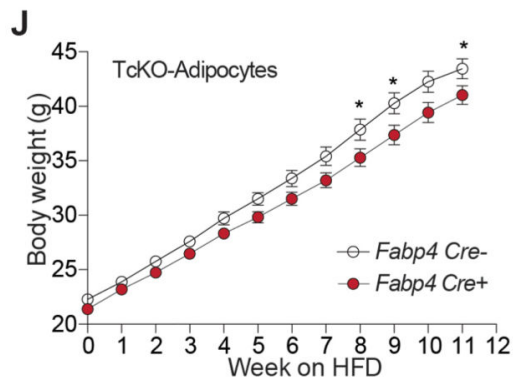

B
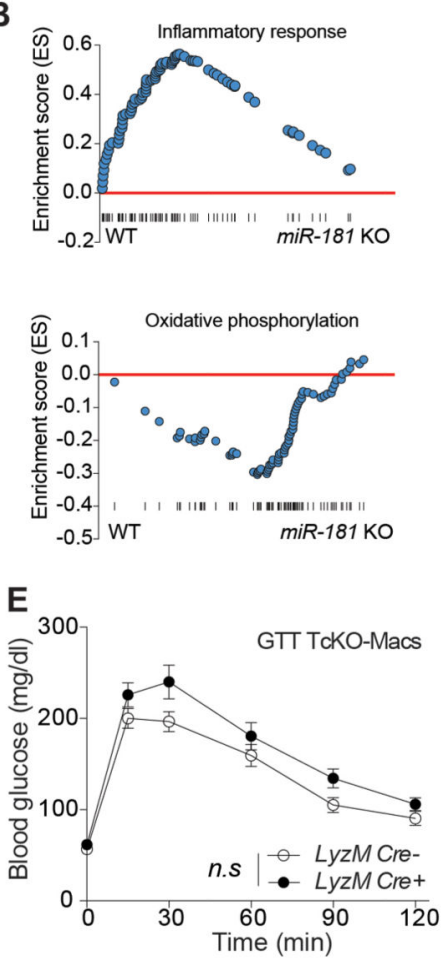

H
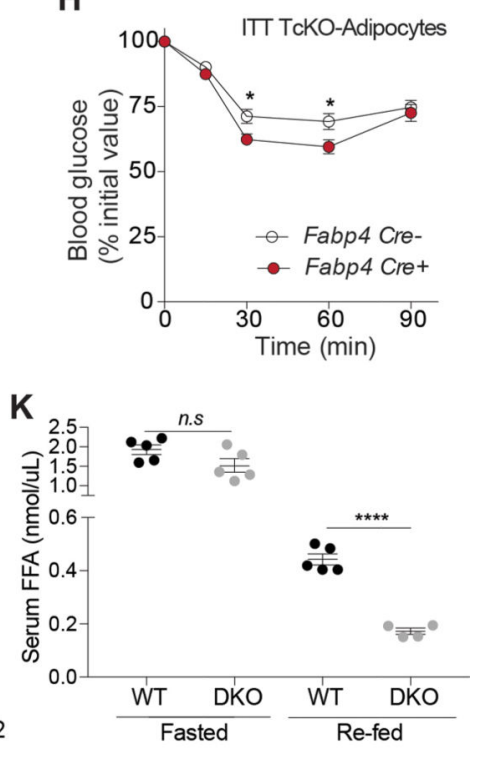

Figure 3. Adipocyte-specific expression of the miR-181 family impacts glucose homeostasis and body weight.

(A-B) RNA sequencing analysis of eWAT from HFD-fed WT and DKO mice. (A) Gene set enrichment analysis (GSEA) of predicted miR-181 targets (normalized enrichment score [NES] -1.11, [ES] -0.24; FDR q 0.32), with heatmap of the top predicted targets that were significantly differentially expressed (FDR <0.05) in WT $(n=4)$ or DKO $(n=4)$ HFD mice. Orange indicates genes implicated in adipose tissue development and function. Pink indicates genes implicated in regulation of or response to insulin signaling. (B) GSEA of 
inflammatory ([NES] 1.54, [ES] -0.57; FDR q 0.26) and oxidative phosphorylation ([NES] -1.09 , [ES] -0.33; FDR q 0.71) gene sets. (C-G) GTT of triple conditional knockout (TcKO) mice fed a HFD from 6 to 18 weeks of age and expressing Cre-recombinase under the control of the following promoters: (C) Cluster of differentiation $4(C D 4)$ promoter (T cells; Cre- n=8, Cre+ n=19, 5 independent experiments), (D) Forkhead box P3 (FoxP3) promoter (Tregs; Cre- n=5, Cre+ n=12, 4 independent experiments), (E) Lysozyme 2 ( $L y z M$ ) promoter (macrophages; Cre- $\mathrm{n}=8, \mathrm{Cre}+\mathrm{n}=8,2$ independent experiments), (F) Albumin promoter (hepatocytes; Cre- $\mathrm{n}=8, \mathrm{Cre}+\mathrm{n}=8$ ), or $(\mathbf{G})$ Fatty acid binding protein 4 (Fabp4;) promoter (adipocytes; Cre- $\mathrm{n}=11, \mathrm{Cre}+\mathrm{n}=10,2$ independent experiments). (H) ITT of Fabp4 TcKO mice fed a HFD from 6 to 18 weeks of age; Fabp4: Cre- n=24 Cre+ n=29, 3 independent experiments. (I) AUC of data from $(\mathrm{G})$ and $(\mathrm{H})$. (J) Body weights of Fabp4 TcKO mice fed a HFD from 6 to 18 weeks of age (Fabp4 Cre- n=11, Fabp 4 Cre+ n=17, 4 independent experiments). (K) Quantification of adipocyte-specific insulin sensitivity via suppression of free fatty acid (FFA) production during fasting-refeeding. NCD-fed WT $(\mathrm{n}=5)$ and DKO mice $(\mathrm{n}=4-5)$ were fasted for $18 \mathrm{~h}$, then given NCD ad libitum for $4 \mathrm{~h}$. Serum FFAs were measured after fasting and refeeding periods. One dot per mouse. Error bars indicate mean \pm SEM. Two-tailed Student's $t$-test $(\mathbf{I}, \mathbf{K})$, or two-way ANOVA $(\mathbf{C}-\mathbf{H}, \mathbf{J}),{ }^{*} \mathrm{p}<$ $0.05,{ }^{* * * *} \mathrm{p}<0.0001$ 

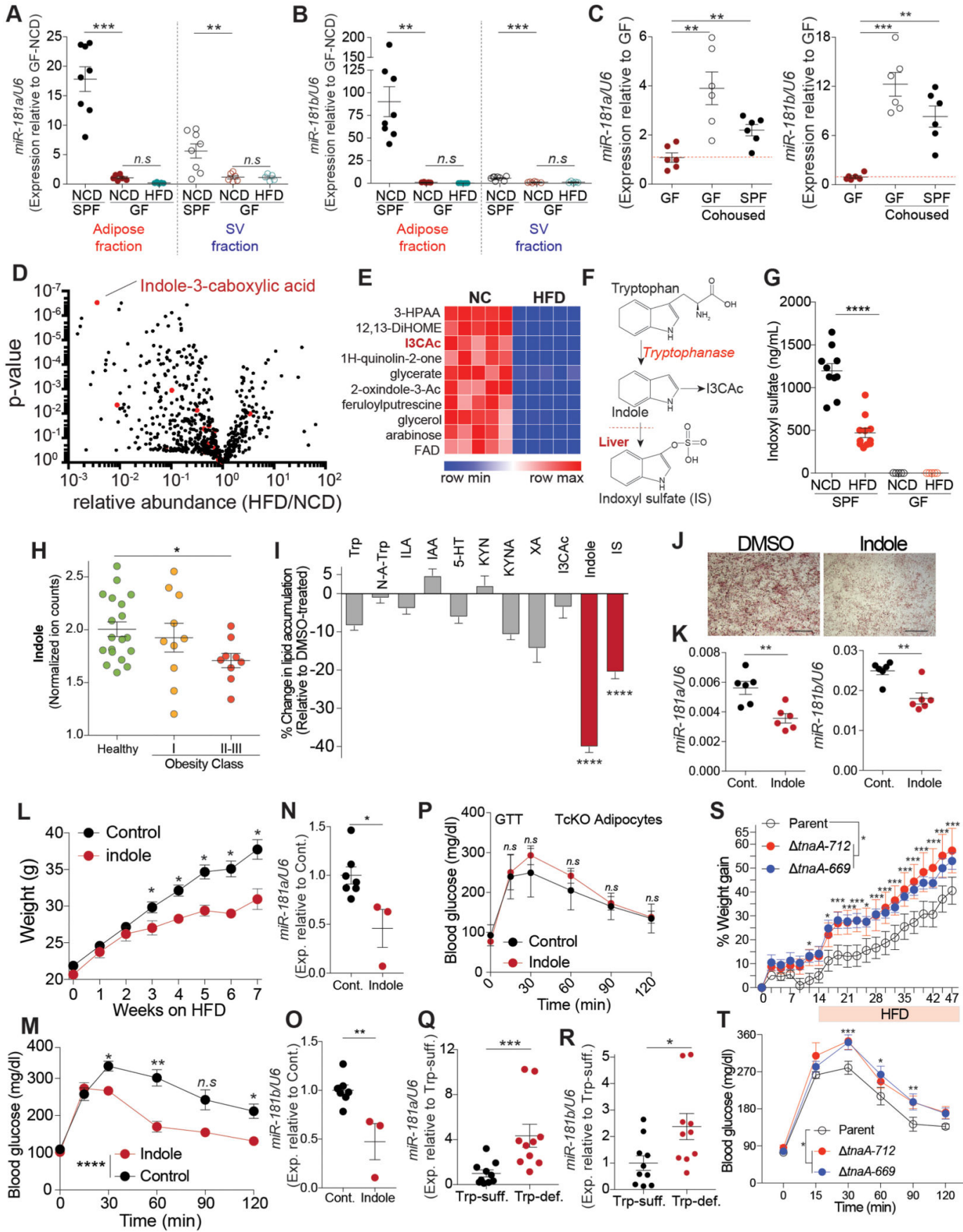

Figure 4. Microbiota-derived metabolites regulate the $m i R-181$ family in white adipocytes to control progression to obesity.

$(\mathbf{A}, \mathbf{B})$ Expression of mature (A) $m i R-181 a$ or (B) $m i R-181 b$ from adipocyte and stromalvascular fractions of eWAT from specific pathogen-free (SPF) mice fed a NCD and germfree (GF) mice fed a NCD or HFD. Expression was normalized to $U 6$ and is shown as fold change relative to NCD GF (NCD SPF n=8, NCD GF n=6, HFD GF n=6, two independent experiments). (C) Expression of mature miR-181a or miR-181b from the adipocyte fraction of eWAT of GF mice $(n=6)$ and cohoused GF $(n=6)$ and SPF $(n=6)$ mice for 8 weeks. 
Expression was normalized to $U 6$ and is shown as fold change relative to GF. (D) Volcano plot of metabolite abundance in the cecal contents of NCD- or HFD-fed mice. Plotted as relative abundance in HFD compared to NCD mice. Metabolites related to tryptophan metabolism are plotted in red. (E) Heat map of the 10 metabolites from (D) most significantly altered in HFD-fed mice compared to NCD-fed mice. (F) Schematic of the conversion of tryptophan to indole, indole-3-carboxylic acid, and indoxyl sulfate showing the role of tryptophanase. (G) Concentrations of indoxyl sulfate determined by mass spectrometry in plasma from 7-9-week-old SPF and GF mice fed a NCD or HFD for 5 weeks. (H) Abundance of plasma indole determined by mass spectrometry from children stratified by obesity status. Using BMI percentiles for age and gender, individuals were binned into healthy weight ( $<85$ th percentile, $n=19)$, class I obesity $(100-120 \%$ of 95 th percentile, $n=10$ ), class II obesity (120-140\% of 95 th percentile, $n=6)$ and class III obesity ( $>140 \%$ of 95 th percentile, $n=3$ ). (I) Lipid accumulation in cultured adipocytes following 6day treatment with tryptophan-derived metabolites $(200 \mu \mathrm{M})$. Plotted as percentage change in Oil Red O staining in metabolite-treated relative to DMSO-treated cells. 4 independent experiments, 3-6 biological replicates per group. $\operatorname{Trp}=$ tryptophan, N-A-Trp $=\mathrm{N}$-acetyltryptophan, ILA = indole lactic acid, IAA = indole acetic acid, 5-HT = 5-hydroxytryptamine (serotonin), KYN = kynurenine, KYNA = kynurenic acid, XA = xanthurenate, I3CA = indole-3-carboxylic acid, IS = indoxyl sulfate. (J) Representative image of cultured adipocytes treated with DMSO or indole $(200 \mu \mathrm{M})$ and stained with Oil Red O. Scale bar, 2.5 $\mathrm{mM}$. (K) Expression of mature miR-181a and miR-181b in differentiated 3T3-L1 cells following 2-day treatment with indole $(100 \mu \mathrm{M})$. (L) Body weight and (M) GTT of HFD-fed mice i.p. injected with indole $(50 \mathrm{mg} / \mathrm{kg} ; \mathrm{n}=4-5)$ or solvent control $(\mathrm{n}=7)$. Weights measured 3 times/week and pooled. (N, O) Expression of mature (N) miR-181a or (O) miR-181b from adipocyte fractions of eWAT from mice in $(\mathrm{L}, \mathrm{M})$, normalized to $U 6$ and shown as fold change relative to solvent-injected mice (one dot per mouse). (P) GTT of miR-181 TcKO Fabp4 HFD-fed mice i.p. injected with indole $(50 \mathrm{mg} / \mathrm{kg}$; $\mathrm{n}=3$ ) or solvent control (n=3) 3 times per week for 7 weeks. $(\mathbf{Q}, \mathbf{R})$ Expression of mature $m i R-181 a(\mathbf{Q})$ or $m i R-181 b(\mathbf{R})$ from adipocyte fractions of eWAT of SPF mice maintained fed a tryptophan-sufficient (Trpsuff., $\mathrm{n}=10$ ) or tryptophan-deficient (Trp-def., $\mathrm{n}=10$ ) diet. Expression was normalized to U6 and is shown as fold change relative to Trp-suff. diet; two independent experiments. (S) Body weight and (T) GTT of mice colonized with a WT parental Escherichia coli (E. coli) strain or tryptophanase E. coli knockout (tna $A \mathrm{KO}$ ) (n=4/group). Error bars show mean \pm SEM. Two-tailed Student's $t$-test $(\mathbf{G}, \mathbf{I}, \mathbf{N}, \mathbf{O}, \mathbf{R})$, one-way ANOVA (A, B, C, H), two-way ANOVA (L, M, P, S, T), or Mann-Whitney test $(\mathbf{K}, \mathbf{Q}){ }^{*} \mathrm{p}<0.05,{ }^{* *} \mathrm{p}<0.01,{ }^{* * *} \mathrm{p}<0.001$ **** $\mathrm{p}<0.0001$. 